\title{
Importancia de los productos agrícolas del grupo hortofrutícola en la alimentación de poblaciones vulnerables
}

Importance of the agricultural products of the horticultural group in the feeding of vulnerable populations

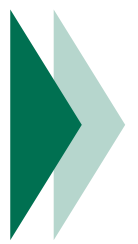

\section{Blanca Rosa Restrepo de Peña}

Economista, Universidad de Santo Tomas. Magister en Educación, Universidad Santo Tomas. Docente-Investigador del grupo Gestión en Ciencia, Innovación, Tecnologías y Organizaciones.

E-mail: blanca.restrepo@ustadistancia.edu.co

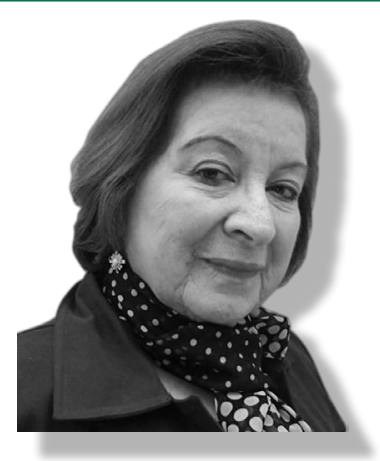


José Elvis Sierra Borrás

Economista Agrícola. Universidad Inca de Colombia. Magister en Planeación y Desarrollo socio económico. Universidad Santo Tomás. Docente-Investigador del grupo Gestión en Ciencia, Innovación, Tecnologías y Organizaciones.

E-mail: josesierra@ustadistancia.edu.co

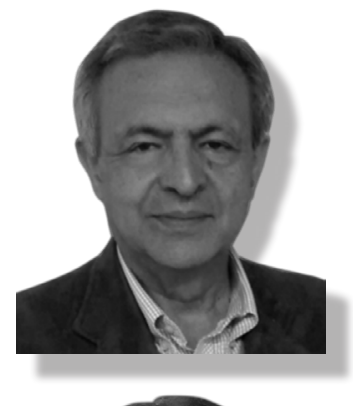

Nubia Esperanza Suárez Suárez

Ingeniero Agrónomo Universidad Nacional de Colombia, MBA executive, Escuela Europea de Negocios Docente-Investigador del grupo Gestión en Ciencia, Innovación, Tecnologías y Organizaciones.

E-mail: nubiasuarez@ustadistancia.edu.co

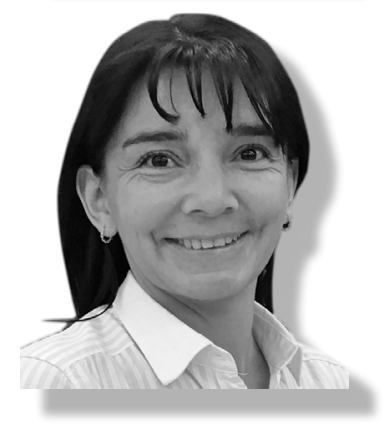

\section{RESUMEN}

Este artículo presenta resultados de un proyecto productivo en agricultura urbana para cubrir necesidades alimentarias y mejorar los ingresos familiares. El estudio descriptivo e interpretativo, se adelantó mediante la investigación Acción Participativa, con muestra al azar de 62 familias, y encuesta estructurada para caracterizar social y económicamente las familias ubicadas en el barrio Loma Linda en la Comuna IV, Altos de Cazucá en el municipio de Soacha. La información se obtuvo de manera participativa, mediante talleres interactivos, el diálogo y la observación. Los resultados, identifican una población de pobreza extrema, con ingresos promedio de $\$ 252.000$ pesos por familia, con prevalencia del adulto mayor de 60 o más años en un $49.9 \%$, educación primaria del 70\% y una tasa de analfabetismo del 30\%. Se analizaron los componentes hortofrutícolas, básicos de alimentación de la población, susceptibles de obtener a través de la agricultura urbana. El análisis evidenció un abastecimiento de estos productos en un 50\% que se pueden producir en la comunidad utilizando sus propios recursos en forma rudimentaria, pero eficiente. Se desarrolló un programa de capacitación que contemplo dos enfoques, el comunitario, logrando la motivación e interés con valores de convivencia y trabajo cooperativo. La capacitación en agricultura urbana enfocada a sistemas y procedimientos de la producción urbana. Fortalecida la población vulnerable iniciaron los cultivos hortofrutícolas, con innovación creatividad y empoderamiento comunitario utilizando los espacios de las viviendas, para cultivar en diferentes formas y recipientes reciclables, se obtuvo productos del consumo familiar. Paralelamente iniciaron cultivo cooperativo para obtener excedentes comercializables en un lote comunitario.

Palabras Clave: Agricultura urbana, productos hortofrutícolas, población vulnerable, seguridad alimentaria, educación comunitaria. 


\section{Abstract}

A productive project was proposed in urban agriculture to cover food needs and to improve family income. The descriptive and interpretativestudywas carried out through the Participatory Action research, with a random sample of 62 families, and a structured survey to characterize socially and economically the families located in the neighborhood Loma Linda in the Commune IV, Altos de Cazucá in The municipality of Soacha. The information was obtained in a participatory manner, through interactive workshops, dialogue and observation. The results identify a population of extreme poverty, with an average income of $\$ 252,000$ (pesos colombianos) per family, prevalence of the adult over 60 years or more in $49.9 \%$, primary education $70 \%$ and illiteracy 30\%. The horticultural components, basic of feeding of the population, were analyzed, susceptible to obtain through with urban agriculture. The analysis showed a 50\% supply of these products that can be produced in the community using their own resources in a rudimentary but efficient way. A training program was developed that contemplated two approaches, the community, achieving the motivation and interest with values of coexistence and cooperative work. Training in urban agriculture focused on systems and procedures of urban production. Strengthened the vulnerable population began horticultural crops, with innovation creativity and community empowerment using the spaces of the houses, to grow in different forms and recyclable containers, products were obtained from family consumption. At the same time they began cooperative cultivation to obtain surpluses marketable in community lots.

Keywords: Urban agriculture, fruit and vegetable products, vulnerable population, food security, community education.

\section{Introducción}

El municipio de Soacha se encuentra ubicado en la zona sur oriente de la sabana de Bogotá, colindando con el Distrito Capital, y es uno de los más extensos del departamento de Cundinamarca. Altos de Cazucá alberga un alto número de población desplazada, tanto por ser oriunda de las zonas de conflicto, como por fenómenos naturales, la cual se localiza en los cerros orientales del municipio.

El Acuerdo No 46 de diciembre 27 de 2000, (exposición de motivos, P.1), que aprueba el POT, afirma que el asentamiento poblacional en condiciones de desplazamiento, en las últimas tres (3) décadas ejerce presiones, que ha venido afrontando, por demanda del suelo barato con el fin de obtener asentamientos para su grupo familiar, el cual se encuentra en condiciones de vulnerabilidad, por lo que han ocupado de manera indebida parte del territorio municipal, el cual presenta la densidad poblacional más alta del país.

Esta invasión ilegal, ha ejercido una fuerte presión sobre el medio natural, propiciando una degradación progresiva de los sistemas naturales de los suelos del municipio, que presentan fragilidad ante los procesos de deterioro, escasez de suelos de calidad y baja capacidad de acogida, que no permiten ser un soporte esencial de actividades de producción agropecuaria.

La investigación se enfocó hacia la población vulnerable ubicada en la Comuna IV Altos de Cazucá, barrio Loma Linda del municipio de Soacha, en la que se concentran numerosas familias de escasos recursos económicos, que no poseen los medios para satisfacer las necesidades básicas en alimentos, especialmente los de origen agrícola como fuente primaria de nutrición (hortalizas, tubérculos, frutas, legumbres, entre otros). De otra parte, estos productos presentan 
estacionalidad de oferta en algunas épocas del año, que ocasionan escasez y en consecuencia precios altos, haciéndolos más inasequibles a estas poblaciones en situación de pobreza extrema e indigencia y, cuya posibilidad son los desechos alimentarios, que logran obtener a través del rebusque en los desechos de Corabastos y otras plazas de mercado.

Con la investigación se unen dos programas de la Facultad de Ciencias y Tecnologías de la VUAD, el programa de Administración de Empresas y el de Administración de Empresas Agropecuarias, que integran un grupo interdisciplinario y el semillero de investigación de Empresas Agropecuarias para implementar los aspectos técnicos de la agricultura urbana, caracterizados con sistemas de siembra, sostenimiento de los cultivos, producción y cosecha. El programa de Administración de Empresas orientó los aspectos organizacionales, enfatizando en valores y principios de convivencia para la cooperación como equipos de trabajo y actividades cooperativas para la producción, con la participación activa de la comunidad local.

El proyecto contribuye a la extensión de catedra mediante las funciones sustantivas institucionales aprovechando la presencia de la universidad con el centro de proyección social Santo Domingo, coordinando acciones con la Fundación Revivir Siglo XXI, que trabaja por la integración de la comunidad, desarrollo de actividades académicas y la aplicación de las técnicas agrícolas en predios de la comunidad. Estas experiencias enriquecen los currículos de los programas académicos con sentido social y humano.

Con estos referentes se formuló la siguiente pregunta de investigación:

¿Cuáles son los productos agrícolas del grupo hortofrutícola que conforman la base de la alimentación en la población vulnerable de la Comuna IV, Altos de Cazucá, municipio de Soacha, y las formas de generar actividades de producción de agricultura urbana con los recursos disponibles en la comunidad?

\section{Objetivo general}

Establecer cuáles son los productos agrícolas, del componente hortofrutícola, que conforman la base de la alimentación en la población vulnerable de la Comuna IV, Altos de Cazucá, municipio de Soacha, y las formas de generar actividades de producción de agricultura urbana con los recursos disponibles en la comunidad.

\section{Objetivos específicos}

- Identificar los productos agrícolas del grupo hortofrutícolas base de la alimentación en la población vulnerable. - Seleccionar los productos agrícolas del grupo hortofrutícola, susceptibles de producir bajo las condiciones de la población

- Establecer la disponibilidad de recursos físicos, humanos y económicos de la población vulnerable para el desarrollo de proyectos de agricultura urbana del grupo hortofrutícola.

- Capacitar a la comunidad en formas de asociación y cooperación para producir los alimentos de origen hortofrutícola seleccionados.

\section{Referentes conceptuales}

\section{Agricultura urbana}

Al referirnos a la agricultura urbana es necesario pensar en todas las formas de 
producción agrícola que se llevan a cabo en el contexto urbano. Cuando se aplica en comunidades vulnerables, su práctica se debe referir desde materas o recipientes colgadas en muros de patios, interiores o ventanas, que se destinan en muchos casos al autoconsumo, hasta los cultivos en jardines, lotes o terrenos más grandes que producen excedentes de cosecha para la venta de los mismos.

Teniendo en cuenta los conceptos expuestos por el Jardín Botánico José Celestino Mutis (2007), la agricultura urbana se refiere a:

El sistema de producción agrícola que se realiza en áreas urbanas como zonas blandas (antejardines o lotes) y zonas duras (terrazas, patios) [u otros espacios] de las que se dispone en la vivienda y su entorno. Es una práctica agrícola que se adelanta en espacios urbanos dentro de la ciudad, o en sus alrededores, utilizando el potencial local como la fuerza de trabajo, el agua disponible, el agua lluvia, los residuos sólidos, integrando los saberes y conocimientos de la comunidad, con el fin de promover la sostenibilidad ambiental y generar productos alimenticios limpios para el autoconsumo y excedentes comercializables para el fortalecimiento del tejido social. (p.1)

El Jardín Botánico complementa en Colombia la política del Programa de Naciones Unidas a través de la Organización para la Agricultura y la Alimentación FAO, para asegurar la alimentación y nutrición de poblaciones pobres y vulnerables mediante la construcción de tejido social, la participación comunitaria, la apropiación del territorio para el desarrollo de proyectos de agricultura. Cantor (2010), citado por Gómez (2014), define la agricultura urbana como la práctica agrícola y pecuaria en áreas intra y peri urbanas de las ciudades, que por iniciativa de los productores, afincados muchas veces en los barrios marginales (favelas, rancherías, barriadas, barrios subnormales), utilizan los mismos recursos locales, como mano de obra, espacios, agua y desechos sólidos orgánicos, con el fin de generar productos de autoconsumo y también destinados a la venta en el mercado [más cercano].

Hermi-Zaar (2011), señala el reciente uso del término agricultura urbana, utilizado en documentos on-line o en textos publicados por la Organización de las Naciones Unidas para la Agricultura y la Alimentación-FAO, con el que se refiere a "prácticas agrícolas que se llevan dentro de los límites o en los alrededores de las ciudades de todo el mundo e incluye la producción, y en algunos casos el procesamiento de productos agropecuarios, pesqueros y forestales“.

La Organización de las Naciones Unidas para el Desarrollo (1999), se refiere a las prácticas agrícolas, dentro de las ciudades y en torno a ellas, [las comunidades] compiten por unos recursos (tierra, agua, energía, mano de obra) que podrían destinarse también a otros fines para satisfacer las necesidades de la población urbana. Este tipo de agricultura se ha venido desarrollando en diferentes países del mundo; se estima que unos 800 millones de habitantes participan en actividades relacionadas con la agricultura urbana y periurbana AUP que generan ingresos $y$ producen alimentos. Una combinación de datos de censos nacionales, encuestas por hogares y proyectos de investigación señalan que hasta dos tercios de los hogares urbanos y periurbanos participan en la agricultura con estas características, FAO et al. (1999) Una gran parte de los productos de la agricultura urbana se destinan al consumo propio, mientras que los excedentes ocasionales se venden en el mercado local.

En Colombia y especialmente en Bogotá, la agricultura urbana se inicia en la década de los años 90, como consecuencia de los desplazamientos de la población rural hacia las ciudades, ocasionada por los conflictos entre grupos armados y la violencia en los campos, 
cuya presión sobre los más humildes, haría que se vieran obligados a dejar sus unidades de producción y asentarse en espacios de invasión en la periferia de las grandes ciudades, en busca de nuevas oportunidades para su subsistencia, como lo describe Marulanda (2002, pp. 37-38). En este sentido, las poblaciones desplazadas, reconocidas también como vulnerables, independientemente de su origen, y ante la imposibilidad de conservar su cultura productiva agrícola, desarrollan formas de producción, acondicionadas a los espacios y recursos disponibles. Es así, como esta población recrea su entorno, iniciando con una agricultura ornamental, hortícola y de especies menores como aves de corral, entre otros, liderado especialmente por las mujeres y posteriormente con la vinculación de hombres del componente familiar, especialmente de la tercera edad.

En Colombia, el programa de Naciones Unidas para el Desarrollo-PNUD, promovió la hidroponía popular a finales de la década de los años 80, con el proyecto regional para la superación de la pobreza de América Latina y del Caribe, siendo Bogotá una de la ciudades donde se estableció esta técnica, en el sector conocido como Ciudad Bolívar, Barrio Jerusalén, y posteriormente se extendió a otras ciudades de Colombia, de América Latina y del África

La misma FAO (1999), señala que la productividad agrícola, sobre todo de hortalizas, obtenida en pequeñas parcelas con tecnologías apropiadas a las condiciones agroecológicas del medio urbano, tiene un rendimiento alto y responde de inmediato a la necesidad urgente de alimentos propios de la comunidad. En estas condiciones, la productividad puede ser hasta 15 veces superior a la obtenida en la agricultura rural. Estudios de la FAO revelan que "un micro-huerto de un metro cuadrado puede producir cualquiera de las siguientes cosechas: unos 200 tomates (30kg) al año; 36 piezas de lechuga cada 60 días; 10 coles cada 90 días; 100 cebollas cada 120 días" (Hermi- Zaar, 2011)

Según Burgos (2007), se afirma que:

La agricultura en zonas urbanas puede ayudar a mejorar la seguridad alimentaria de diversas formas: producir alimentos en casa o a través de [terrenos localizados en la comunidad], la integración de superficies colindantes, terrazas, o huertos caseros en áreas comunes], reduce el gasto para las familias, pone más alimentos a su alcance y reduce la escasez de productos frescos. (p.8)

Situación que es viable para mejorar las condiciones de vida del $77 \%$ de la población más pobre en Cazucá.

Igualmente, el estudio afirma que ha sido política de los diferentes gobiernos, y de interés permanente de organismos internacionales como FAO, Organización de Naciones UnidasONU, y la Comisión Económica para América Latina-CEPAL (ROBLES, 2002), entre otros, de fomentar la agricultura urbana para mitigar las diferencias alimentarias de las poblaciones pobres y vulnerables de los países Latinoamericanos en desarrollo, además de generar excedentes comercializables para obtener ingresos familiares.

\section{Productos hortofrutícolas}

Los productos hortofrutícolas son alimentos básicos en la dieta humana, pero tienen el inconveniente de ser perecederos, por lo que se dispone de ellos durante períodos cortos de tiempo, siendo además en muchos casos cultivos de carácter estacionario. Sin embargo estos productos son viables para mejorar las condiciones de vida del $77 \%$ de población más pobre de los estratos 0,1 y 2, en Altos de Cazucá, al producir los productos hortofrutícola básico de la alimentación, los cuales, según presentación y clasificación de la Fundación Eroski (2016), se mencionan los siguientes: 
1. Brasicáceas: brócoli, coliflor, nabo y rábano

2. Cucurbitáceas: calabacín, calabaza y pepino

3. Fabáceas: haba, arveja y fríjol verde

4. Gramínea: maíz

5. Hortaliza de hoja verde: acelga, apio, espinaca, lechuga y perejil

6. Liliáceas: ajo y cebolla

7. Quenopodiáceas: acelga, espinaca y remolacha

8. Solanáceas: berenjena y tomate

9. Umbelíferas: apio, perejil y zanahoria.

\section{Alimentación y nutrición familiar}

Teniendo en cuenta las orientaciones de Burgers \& Glasauer (2006), consultores de la FAO afirman:

La pobreza es la mayor causa de los problemas nutricionales encontrados en los países en desarrollo. Pero la malnutrición también existe donde la población no es pobre o donde las personas pueden disponer de lo suficiente para comer. Esto se manifiesta claramente en el hecho de que existan dos [tipos de nutrición] - bastante opuestos. El primero es el resultado de una ingesta insuficiente de alimentos saludables e inocuos. El segundo es causado por una ingesta excesiva o desequilibrada de alimentos o de ciertos tipos de alimentos. Ambas pueden ser prevenidas con una alimentación saludable, que recomienda consumir todos los días frutas, verduras, legumbres, cereales, lácteos y proteínas, entre otros. (p. III)

La alimentación es la base de la salud de las personas, en la medida que proporciona al organismo las sustancias que le son indispensables para la subsistencia. En este sentido, la variedad en los alimentos proporciona los nutrientes necesarios, debido a que no existe uno que contenga todos los nutrientes para el organismo, cada alimento contribuye de manera específica, de acuerdo a sus componentes. Por lo tanto, las familias necesitan suficientes recursos para producir y/o comprar los alimentos necesarios. También es importante capacitarlos para que puedan entender qué "combinaciones de alimentos constituyen una alimentación saludable y tener las habilidades y motivación necesarias para adoptar decisiones apropiadas sobre el cuidado de la familia y las prácticas alimentarias" (Burgers \& Glasauer, 2006) que constituyen finalidades propias de la agricultura urbana.

Desde finales del siglo XX, y generalmente apoyados por la FAO, el número de países subdesarrollados donde se han promovido experiencias relacionadas con la agricultura urbana se ha incrementado. Con la finalidad de solucionar la carencia alimentaria, han sido los países que poseen poblaciones con mayor riesgo de inseguridad alimentaria los que, a partir de la década de 1990, han desarrollado algún tipo de normativa pública de incentivos para la agricultura urbana, tanto en las grandes ciudades como en otras poblaciones menores.

Adam Prakash (2010) de la División de Comercio y Mercados, de la FAO, investigó la importancia y prevalencia de la agricultura urbana y sus repercusiones en la seguridad de las familias, en una muestra de 15 países en desarrollo y formalmente en transición. Los resultados indican que la horticultura es una actividad significativa en la economía urbana, participa un $65 \%$ de las familias urbanas.

\section{Seguridad alimentaria}

Según la Cumbre Mundial sobre la Alimentación de 1996, citado por Mosquera (2009) afirma que:

Existe seguridad alimentaria cuando todas las personas tienen en todo momento acceso físico y económico a suficientes alimentos inocuos y nutritivos para satisfacer sus necesidades alimenticias y sus preferencias en 
cuanto a los alimentos a fin de llevar una vida activa y sana. (p.41)

Este criterio señala las siguientes dimensiones de la seguridad alimentaria: disponibilidad de alimentos y acceso a los mismos en todo momento. Sin embargo, se evidencia que en muchas poblaciones, se presenta carencia de disponibilidad y acceso a los alimentos básicos que garanticen la seguridad alimentaria, en forma adecuada, suficiente y oportuna en el tiempo, especialmente en los que se encuentran en condiciones de vulnerabilidad o extrema pobreza, bien porque no tienen recursos o ingresos suficientes para adquirirlos, o que el estado es ausente en el aprovisionamiento de los mismos, o las condiciones de producción no se ajustan a las necesidades de estas.

La agricultura urbana, se constituye en parte de la solución, debido a que la misma población puede producir sus propios alimentos con sus propios recursos dando lugar a la oportunidad y suficiencia de la producción de acuerdo a sus necesidades y con características de una dieta más diversificada y un aumento en el consumo relativo a precios accesibles o razonables.

También se encuentra seguridad alimentaria cuando las personas tienen acceso a los alimentos suficientes, inocuos y nutritivos que satisfagan sus necesidades energéticas diarias y preferencias alimentarias para llevar una vida sana y activa, como lo describen Brito, Betancour, \& Hernández (2012).

Las distintas formas de organizar las actividades productivas se plasman en el método utilizado, que a su vez vendrá condicionado a la naturaleza de la organización de la familia, individual o mediante colectivos productivos. Las reflexiones de Hermi- Zaar (2011), aseguran que:

Si bien la agricultura es en su mayor parte un fenómeno rural, la agricultura urbana puede ayudar a incrementar la capacidad de resistencia a los impactos externos de parte de la población urbana pobre y mejorar su acceso a las frutas y hortalizas frescas y a los productos animales. Este mecanismo tendrá particular importancia en zonas en las que la infraestructura inadecuada y las elevadas pérdidas durante el transporte se añaden a la escasez y alto costo de los productos agrícolas. Algunos agricultores urbanos podrían además ofrecer sus productos en los mercados locales, generando ingresos para ellos mismos y sus familias.

En el anterior contexto, la agricultura urbana se constituye en una alternativa de producción para mejorar la calidad de vida de las poblaciones vulnerables, al disponer de alimentos que favorecen la seguridad alimentaria y por lo tanto, una salud apropiada a sus condiciones de vida y a su nivel de ingresos.

\section{Organización comunitaria}

Montero (2004, citado por Sierra y Peña, 2014) hace referencia a la comunidad como:

Un grupo social dinámico, histórico y culturalmente constituido y desarrollado, preexistentealapresencia de losinvestigadores o de los interventores sociales, que comparte intereses, objetivos, necesidades y problemas, en un espacio y un tiempo determinado y que genera colectivamente una identidad, así como formas organizativas, desarrollando y empleando recursos para lograr sus fines. (p.17)

Bajo esta concepción, los grupos identificados en Altos de Cazucá, se ajustan a estas características, por cuanto su origen de inmigrantes de zonas rurales de varios zonas o departamentos del país, convergen en manifestar necesidades y objetivos afines, con el interés de plantear acciones de solución a los problemas comunes, comprometiendo sus propios recursos para lograr un bienestar 
social generalizado, con sentido de pertenencia hacia los miembros que la constituyen.

Lo anterior, implica implementar acciones o actividades en el que predomina la importancia de un trabajo colectivo y cooperado, en el cual todos asumen responsabilidades y compromisos en forma voluntaria y desinteresada, de tal forma que, en el desarrollo de las interacciones humanas, surge la asociatividad como factor integrador para la organización de la comunidad. El resultado de este proceso beneficia al colectivo, la unidad de criterios y objetivos claros para pensar y actuar, traza la línea de acción para la obtención de metas comunes. Si bien la comunidad es consciente de asumir su propio bienestar mediante la unión de esfuerzos, implica una preparación que les permita conocer, reflexionar, analizar y decidir sobre la conveniencia o no de la integración que conlleve al reconocimiento de su situación actual y la autonomía para participar decididamente en la necesidad de definir las acciones a seguir, para lograr su propio bienestar.

\section{Educación solidaria}

Uno de los aspectos del desarrollo comunitario es la preparación de las personas interesadas en organizar actividades de bienes y servicios, dentro del marco de la educación solidaria, según la Ley 454 de 1998, de la Economía Solidaria, como el proceso permanente orientado a fortalecer la práctica de valores de solidaridad, cooperación y ayuda mutua, con el fin de generar actitudes y conductas que permitan consolidar un compromiso social de trabajo, una convivencia con responsabilidad social, el ejercicio de la democracia participativa para la gestión y toma de decisiones, que les facilite concretar ideas innovadoras y emprendedores encaminadas al desarrollo de proyectos sociales en beneficio de la comunidad.
En este contexto, la educación para la solidaridad se asume en la propuesta a través de varios núcleos temáticos tales como son: asociatividad, emprendimiento, relaciones humanas solidarias, alteridad, autogestión, trabajo en equipo y comunicaciones, ética $\mathrm{u}$ honestidad personal y social, entre otros, como lo enuncia Siabato (2009).

Así mismo, se tienen en cuenta las consideraciones de Ramírez (1989), al citar a Lars Marcus al referirse a los valores de:

Valores de autoayuda: actividad creatividad, autonomía, responsabilidad e independencia. Valores de ayuda mutua: cooperación, unidad de acción colectiva, solidaridad, paz. Valores sin ánimo de lucro: conservación de recursos, [generación de excedentes para el colectivo], responsabilidad social, metas útiles. Sin aprovecharse del trabajo del otro. Valores de educación: conocimiento, comprensión, discernimiento [y toma de decisiones]. Valores de propósito, servicios de calidad y beneficios para los asociados Valores de universalidad: perspectivas globales, franqueza [compromiso y comprensión]. (p.136).

Con estas bases se adelantó la programación delaeducaciónsolidaria, quesefundamentanen las siguientes características: ser participativa para estimular el pensamiento crítico y reflexivo, la cooperación, la creatividad, la cohesión social, la innovación, la convivencia, el respeto a la diferencia y a la expresión libre, la democracia, afectividad y la identidad cultural, temas que se abordan con la misma comunidad en un proceso de construcción, guiada por los orientadores o facilitadores en interacción con misma. Este método de la educación solidaria, no es ajeno a la dinámica que establece la IAP, en la que en interacción comunidad-investigadores, permite en un principio identificar problemáticas, formular e implementar acciones de solución, a través de la organización, capacitación y gestión de la comunidad. 


\section{Metodología}

El enfoque metodológico está marcado por la investigación acción participativa, IAP, en el que se integró un grupo de investigación, interdisciplinario de docentes, de dos programas de la Facultad de Ciencias y Tecnologías, Administración de Empresas y Administración de Empresas Agropecuarias de la VUAD y los participantes de la comunidad, quienes integraron un grupo representativo y quienes son los propios gestores del proyecto, protagonistas de las transformaciones de su propia realidad y constructores de su mejoramiento de vida.

La participación de todos los integrantes del grupo, adelantaron un proceso de autoconocimiento, transformación de sí mismo, como también la realidad estudiada de acuerdo con lo expuesto por Bernal (2006), quien enfatiza sobre la comunicación y retroalimentación de procesos prácticos, en el que todos aprenden y cooperan con los demás para el desarrollo de productos. De igual manera, se desarrolla la conciencia autónoma reflexiva y crítica, para el mejoramiento permanente de los procesos. El grupo de facilitadores interdisciplinarios orientan y acompañan la formación y capacitación en los elementos esenciales de organización comunitaria, valores y principios solidarios para la convivencia en cooperación y ayuda mutua y al éxito de las actividades prácticas para la obtención de información, experiencias y resultados exitosos de las acciones en beneficio de la comunidad.

\section{Proceso seguido en la investigación}

Inicialmente se adelantó una fase de acercamiento y reconocimiento de la población, con el coordinador del Centro de Proyección Social de la Universidad Santo
Tomás, en Altos de Cazucá, igualmente con la Asociación de la tercera edad, lugar en la que se convocó la reunión con el propósito de motivar la participación de los representantes de las familias, y se explicó los objetivos de la investigación y la importancia de la participación de estos en el aporte de la información requerida.

Para la recolección de información se diseñó una encuesta estructurada (Ver Anexo No. 1) para la caracterización de la comunidad, en la que se definieron los principales tópicos sociales y económicos en cuanto al componente familiar, nivel educativo, condiciones del hábitat, ingresos, ocupación u oficio, las actividades relacionadas con la agricultura urbana y las experiencias en su lugar de convivencia.

Definición de los bienes primarios de consumo de la población vulnerable de estratos 0,1 y 2, de Altos de Cazucá, enfocados hacia los productos hortofrutícolas de interés para el estudio.

Selección de una muestra estadísticamente representativa, tomada al azar de los integrantes de la población, localizados en el barrio Loma Linda, conformada por 58 familias de las que aportaron información los adultos mayores, de una población total de 420 núcleos familiares residentes en este barrio, según información de la Alcaldía de Soacha y verificada con lo reportado por el DANE, para esta comuna.

La fórmula estadística aplicada para determinar el tamaño de la muestra fue la siguiente:

$$
n=\frac{P Q}{\left(\frac{E}{Z}\right)^{2}+\frac{P Q}{N}}
$$


-N = Tamaño de la población objeto que es de 420 familias

•n = Tamaño de la muestra

•P = Probabilidad de aceptación del 0.90, definida por el equipo investigador

$\bullet \mathrm{Q}=1-\mathrm{P}=0.5$

$\bullet \mathrm{E}=$ Margen de error igual a 0.10

El tamaño de la muestra calculada con la formula anterior, fue de 58 familias. Sin embargo por el interés demostrado por la comunidad, se seleccionaron 62 familias que reportaron información y opiniones solicitadas en el instrumento de recolección de información.

También se llevaron a cabo talleres grupales e interactivos en los que se obtuvo la información de caracterización socio económico y definición de alimentos del grupo hortofrutícolas y la disponibilidad de recursos para la producción de estos bienes. De igual manera y en forma paralela se identificó la intención de la asociatividad y necesidad de formación en valores y principios solidarios. El componente técnico para el establecimiento y manejo de los cultivos en condiciones de agricultura urbana, fueron cubiertos por especialistas agropecuarios del semillero de investigación del Programa de Administración de Empresas Agropecuarias.

\section{Resultados}

De las 62 familias, se obtuvo que están conformadas por un total de 391 personas, para un promedio de 6 personas por familia aproximadamente. De esta población resalta la prevalencia de población del adulto mayor en la que el 49.9\% supera los 61 años en promedio, como se observa en el gráfico No.1, la cual se encuentra en condiciones limitadas de educación, cognitiva, salud y económica.

\section{Grafico No. 1. Distribución de la población por rango de edades.}

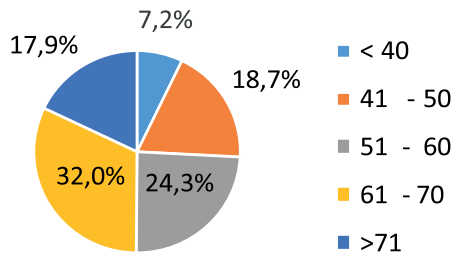

Gráfica 1. Distribución de la población por rango de edades.

Fuente. Elaboración propia. Investigación 2016. Encuesta aplicada a la población.

Resalta el grupo de edad inferiores a 50 años con el $25.9 \%$, que, de acuerdo con la información obtenida, son los que en alguna forma sustentan el grupo familiar, por su vinculación en actividades ocupacionales. Se establece que el promedio ponderado de edad es de 58 años, para la población observada, considerándose una población económicamente activa decreciente.

En cuanto a la distribución de la población por género se obtuvo que el $27.7 \%$ corresponde al género masculino y en mayor proporción con $72.3 \%$ al género femenino. Esta condición demuestra que la mujer prevalece como cabeza de familia y recae la responsabilidad de su sostenimiento y crianza de los hijos.

Respecto a los niveles educativos (gráfico No. 2), el 70\% de la población tiene un nivel primario, el $5 \%$ secundario y el $25 \%$ no ha tenido oportunidad de recibir educación formal, considerándose en condiciones de no saber leer, ni escribir, es decir en situación de analfabetismo funcional. Teniendo en cuenta los rangos de edades se establece que las personas menores de 40 años, el 20\% cuando menos tienen un nivel primario y el 3\% secundario, aunque en este último solamente han llegado hasta el primer año de secundaria. 
Gráfico No. 2 Nivel educativo de la población

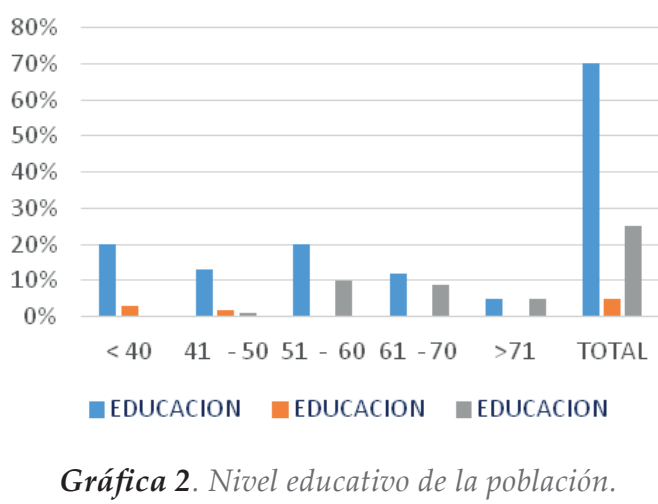

Fuente. Elaboración propia. Investigación 2016. Encuesta aplicada a la población.

El 37\% de la población son mayores de 51 años, solo han cursado la primaria y es el rango de edad que presenta el mayor grado de analfabetismo, situación que es impedimento para obtener calificación ocupacional. En cuanto a la actividad ocupacional, como se observa en la tabla No.1, el 15\% de la población desempeña una actividad laboral como independiente y en igual proporción como dependiente. El 70\% restante no presenta ocupación en ninguna de estas modalidades. La dependencia ocupacional en el $15 \%$ de la población, con edades inferiores a los 60 años, se desempeña en actividades de servicio, como recolectores de basuras, o en otros oficios poco calificados.

\begin{tabular}{|l|l|l|l|}
\hline \multirow{2}{*}{$\begin{array}{l}\text { Rango } \\
\text { de Edad }\end{array}$} & \multicolumn{3}{|c|}{ Actividad ocupacional } \\
\cline { 2 - 4 } & Independiente & Dependiente & Sin vínculo \\
\hline 40 & $10 \%$ & $5 \%$ & $10 \%$ \\
\hline $41-50$ & $3 \%$ & $4 \%$ & $5 \%$ \\
\hline $51-60$ & $2 \%$ & $6 \%$ & $18 \%$ \\
\hline $61-70$ & $0 \%$ & $0 \%$ & $20 \%$ \\
\hline$>71$ & $0 \%$ & $0 \%$ & $17 \%$ \\
\hline TOTAL & $15 \%$ & $15 \%$ & $70 \%$ \\
\hline
\end{tabular}

Tabla 1. Actividad ocupacional de la población.

Fuente. Elaboración propia. Investigación 2016. Encuesta aplicada a la población.
El vínculo laboral como independiente se encuentra en el $15 \%$ de la población con edades inferiores a 60 años, siendo mayor la ocupación en menores de 40 años, con actividades informales en la construcción, recicladores, jardinería, vendedores ambulantes o en oficios domésticos para el caso de las mujeres. El $70 \%$ restante sin vínculo laboral, no significa que tengan actividad productiva permanente, sino que eventualmente o temporalmente desempeñan algún oficio en su entorno o en su propia vivienda generadora de ingresos poco remunerados, derivados del cuidado de niños, que generalmente son de su mismo núcleo familiar (nietos, sobrinos) o hijos de vecinos.

Se encontró que un grupo mayoritario de esta población tienen experiencia en actividades agrícolas por su procedencia de zonas rurales, por lo que se les ha facilitado el desarrollo de este oficio en estructuras de su vivienda mediante materas, recipientes de plástico $\mathrm{u}$ otros sistemas propios de la agricultura urbana, lo que les ha proporcionado una manera de subsistencia. El alto porcentaje sin vínculo laboral, por su bajo nivel educacional o de analfabetismo, no les ha permitido una preparación adecuada en otros oficios.

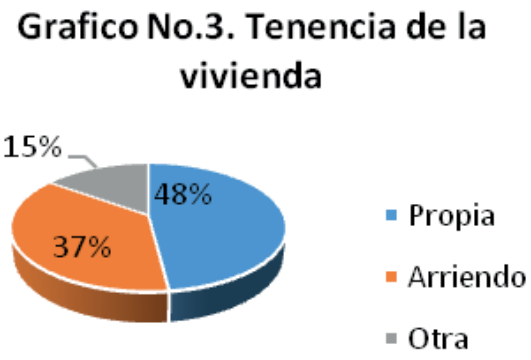

Gráfica 3. Tenencia de la vivienda.

Fuente. Elaboración propia. Investigación 2016. Encuesta aplicada a la población. 
En el gráfico No.3 se observa que el $48 \%$ de la vivienda es propia y en algunos casos sin legalizar, el $37 \%$ en arriendo y el $15 \%$ en otro tipo de posesión como el caso de invasión. Como características generales se establece la situación de invasión en la mayoría de los predios, por desplazamiento forzado de las familias.

En el estudio realizado por Restrepo, Sierra \& Bayona ( 2014), se concluye que “ más del $14 \%$ es población desplazada proveniente de los departamentos de Caquetá, Boyacá, Meta y Cauca, especialmente" (p.154). En el actual estudio, se obtuvo que el porcentaje es mayor, superando el 20\%, debido al desplazamiento de sus lugares de origen por efectos del conflicto armado y la inseguridad rural, lo que les ha obligado a ocupar zonas de Altos de Cazucá, que se califican de alto riesgo, como es el caso del barrio Loma Linda y sus alrededores. También se destaca que estos barrios están dados a la desatención de las autoridades del municipio de Soacha, aduciendo que no hay inversión social por la situación de ilegalidad de los predios ocupados.

En cuanto al nivel de ingresos por familia, se tiene que el $72 \%$ de la población se ubica en el rango entre $\$ 101.000$ y $\$ 400.000$ pesos, para un promedio ponderado de $\$ 252.000$ pesos por mes, con lo que se demuestra que esta zona de Cazucá se encuentra en condiciones de pobreza extrema (Tabla No. 2)

\begin{tabular}{|l|l|}
\hline $\begin{array}{l}\text { Rango de ingresos (Miles } \\
\text { de Pesos) }\end{array}$ & Participación poblacional \\
\hline$<100$ & $15 \%$ \\
\hline $101-200$ & $22 \%$ \\
\hline $201-300$ & $32 \%$ \\
\hline $301-400$ & $18 \%$ \\
\hline
\end{tabular}

\begin{tabular}{|l|l|}
\hline $\begin{array}{l}\text { Rango de ingresos (Miles } \\
\text { de Pesos) }\end{array}$ & Participación poblacional \\
\hline $401-600$ & $8 \%$ \\
\hline $601<$ & $5 \%$ \\
\hline Total & $100 \%$ \\
\hline
\end{tabular}

Tabla 2. Ingresos promedios de la población por rango de edades.

Fuente. Elaboración propia. Investigación 2016. Encuesta aplicada a la población.

De igual manera, se observa en la misma tabla que el $64 \%$ de la población, recibe ingresos inferiores a $\$ 300.000$ pesos mensuales y entre estos el $15 \%$ obtienen menos de \$100.000, comprobándose, las condiciones de pobreza extrema para la población estudiada.

En cuanto a la alimentación básica de la población, está constituida por productos no solamente de origen agropecuario, sino por otros bienes, requeridos para el sustento y complemento alimenticio de la familia, como elementos de aseo e higiene entre otros.

En cuanto al análisis de los productos hortofrutícolas, susceptibles de obtener mediante procesos de agricultura urbana, se evidenció que estos se desarrollan en la infraestructura de las viviendas de los hogares, donde se adelantan de manera artesanal o rudimentaria. 


\begin{tabular}{|c|c|c|c|c|}
\hline Producto & $\begin{array}{l}\text { Cantidad producto } \\
\text { (Lbs.) }\end{array}$ & Vr. Total (\$ pesos) & $\%$ del total & \$/Promedio/unidad \\
\hline Frutas & 12 & 14.467 & $9,4 \%$ & $1.173,0$ \\
\hline Verduras (\# manojos) & 12 & 15.033 & $9,8 \%$ & $1.218,9$ \\
\hline Legumbres & 14 & 13.533 & $8,8 \%$ & 990,2 \\
\hline Hortalizas & 13 & 10.900 & $7,1 \%$ & 860,5 \\
\hline Tubérculos & 44 & 19.400 & $12,6 \%$ & 440,9 \\
\hline $\begin{array}{l}\text { Aromáticas (\# mano- } \\
\text { jos) }\end{array}$ & 3 & 1.500 & $1,0 \%$ & 562,5 \\
\hline Cárnicos & 14 & 60.667 & $39,4 \%$ & $4.439,0$ \\
\hline Huevos & 11 & 3.200 & $2,1 \%$ & 300,0 \\
\hline Leche & 7 & 15.400 & $10,0 \%$ & $2.200,0$ \\
\hline Total & 129 & 154.100 & $100 \%$ & $1.194,6$ \\
\hline
\end{tabular}

Tabla 3. Componentes de alimentación de la población vulnerable.

Fuente. Elaboración propia. Investigación 2016. Encuesta aplicada a la población.

Los productos enunciados en la tabla No. 3 , ascienden a un valor total por mes de $\$$ 154.100 pesos, lo cual equivale a $\$ 25.683$ per cápita, para un núcleo familiar de 6 personas. Este costo indica que cada familia dedica el $61.2 \%$ de sus ingresos para adquirir los productos relacionados en esta tabla, cuando el promedio de la población pobre de Bogotá dedica en promedio el 35\% de sus ingresos según Gómez (2004). Así, se deduce que el $38.8 \%$ restante de los ingresos de la población de Cazucá, lo distribuye en los demás gastos de su subsistencia. No obstante, la salud y educación se suple con los servicios del Estado a través del Sisben y de los establecimientos públicos educativos, respectivamente.

En este contexto de pobreza, la misma comunidad destaca la importancia de desarrollar proyectos de emprendimiento productivo con sus propios recursos, de aquellos bienes queseadapten alas condiciones agroecológicas y de infraestructura, como el caso de las verduras, legumbres, hortalizas, aromáticas y algunos frutales de fácil acceso, como la curuba, fresa, mora y tomate de árbol.

El 65\% de familias son conscientes de la necesidad de desarrollar procesos productivos de agricultura urbana aprovechando sus experiencias. El 25\% de estas manifestaron que no saben si participar o no, por razones de desconocimiento de la actividad, pero demostraron interés de hacerlo, si reciben capacitación en agricultura urbana. El 10\% restante son renuentes por motivos de tiempo y dedicación a otros oficios de trabajo y del hogar. Estos resultados se presentan en la Tabla 4.

\begin{tabular}{|l|l|}
\hline Respuesta & $\%$ \\
\hline Si & 65 \\
\hline No & 10 \\
\hline No sabe & 25 \\
\hline TOTAL & 100 \\
\hline
\end{tabular}

Tabla 4. Participación potencial en la producción de productos Fuente. Elaboración propia. Investigación 2016. Encuesta aplicada a la población. 
También se observó en el grupo, la intención de asociatividad, complementariedad e integración para desarrollar estos proyectos con el propósito de un intercambio de productos mediante la comercialización o trueque directo o en compra venta a precios razonables, que incrementen los ingresos con la venta de los excedentes generado en los mercados locales.

La potencialidad de los huertos a desarrollar en su hábitat por parte de las familias, se presenta en la tabla No.5, en la cual observa la preferencia hacia el cultivo de las verduras (33\%, del a población) con productos como la acelga, espinaca, lechuga, pepino, entre otras. Las hortalizas, legumbres y tubérculos representan el 38\%, con preferencia hacia el tomate, arveja, zanahoria, remolacha, cebolla larga y cabezona, dependiendo de la disponibilidad de espacio físico e infraestructura requerida con que la comunidad cuenta.

\begin{tabular}{|c|c|c|}
\hline GRUPO & NDE SITIOS & $\%$ DETOTAL \\
\hline Hortaliza & 60 & $21 \%$ \\
\hline Legumbre & 41 & $15 \%$ \\
\hline Tubérculo & 5 & $2 \%$ \\
\hline Verduras & 92 & $33 \%$ \\
\hline Aromáticas & 66 & $24 \%$ \\
\hline Frutales & 16 & $6 \%$ \\
\hline TOTAL & 280 & $100 \%$ \\
\hline
\end{tabular}

Tabla 5. Huertos en la producción de productos

Fuente. Elaboración propia. Investigación 2016. Encuesta aplicada a la población.

En la tabla No. 6, se refleja el espacio físico con que cuenta la comunidad para el desarrollo de los proyectos de agricultura urbana. El mayor recurso se ubica con materas de diferentes materiales, representando el $83 \%$ del total, especialmente en materiales reciclables como envases plásticos, llantas, y tarros, entre otros, que no implican costos y se pueden ubicar fijándolos a las paredes interiores de los patios, antejardines; el 18\% restante dispone de patios y solares interiores para la plantación directa en tierra de las especies cultivables que requieren de mayor área como por ejemplo la cebolla, papa, arveja, tomate, zanahoria e inclusive aves de corral, u otros especies menores domésticas. De otra parte, se estableció que la comunidad cuenta con dos (2) lotes localizados en el barrio Loma Linda de Cazucá, con extensión limitada pero apropiada y cultivables de manera cooperada con el fin de producir en mayor escala, cultivos destinados a generar excedentes para el consumo de la misma comunidad o destinados a la comercialización en los mercados locales del barrio.

\begin{tabular}{|l|l|l|}
\hline SISTEMA & $N^{\circ}$ DE SITIOS & \% DETOTAL \\
\hline Matera & 152 & $83 \%$ \\
\hline Patio & 25 & $14 \%$ \\
\hline Solar & 7 & $4 \%$ \\
\hline TOTAL & 184 & $100 \%$ \\
\hline
\end{tabular}

Tabla 6. Sistema de producción.

Fuente. Elaboración propia. Investigación 2016. Encuesta aplicada a la población.

Las características de estos lotes y alguno de los sistemas de producción avícola, se puede observar en la fotografía No. 1 


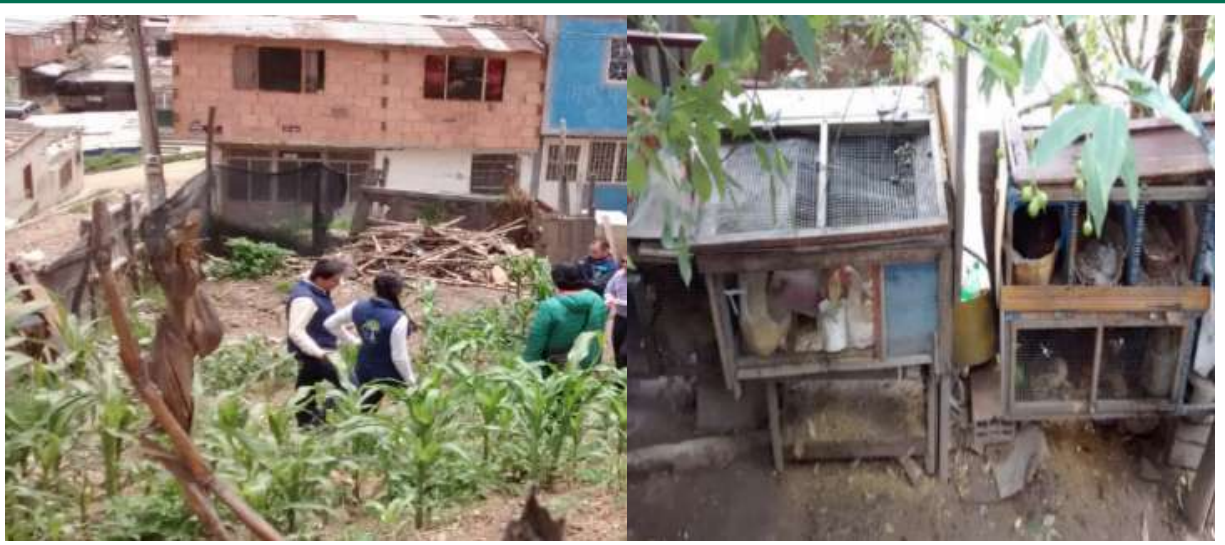

Fotografía 1. Lote cultivable de la comunidad y avicultura urbana.

Fuente. Elaboración propia.

Al consultar a los miembros de la comunidad, sobre su experiencia en procesos de producción de agricultura urbana, el 65\% de estos manifestaron tener alguna experiencia por su procedencia del área rural y actualmente su dedicación en proyectos similares en su hábitat urbano, en pequeña escala, sin técnica apropiada, y apoyándose en la mano de obra familiar (Gráfico No. 4).

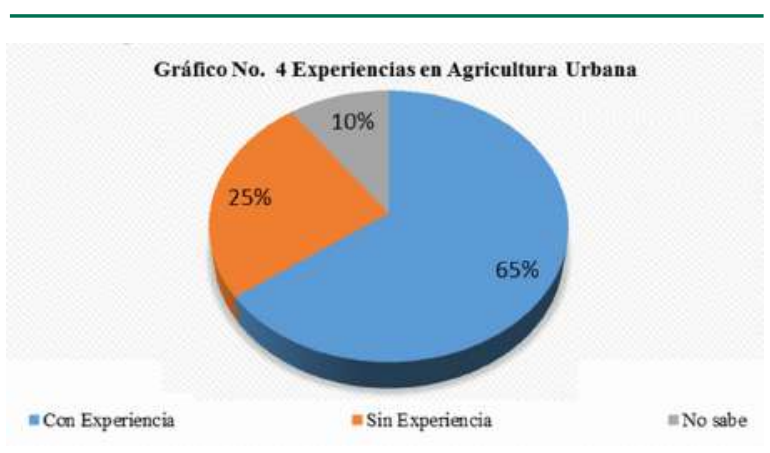

Gr'afico 4. Experiencias en agricultura urbana.

Fuente. Elaboración propia. Investigación 2016. Encuesta aplicada a la población

Se puede observar en el mismo gráfico, que el $25 \%$ de la población no cuenta con experiencia en estos sistemas de producción y el $10 \%$ no lo conocen, pero manifiestan su interés en vincularse al programa de producción de agricultura urbana.
De igual manera establecieron la condición de la presencia de la Universidad para recibir formación y capacitación en tales temas.

En cuanto a la formación en organización comunitaria se desarrolla en diferentes ámbitos que integran la convivencia, en la que se adelantan lazos de amistad, solidaridad e interacción para lograr un bienestar común. Estos procesos no solamente se realizan a través de un diagnóstico, sino que requieren acciones de apoyo y acompañamiento permanente para el logro de cambios y mejoramiento en sus niveles de vida.

Los promotores o facilitadores que acompañaron en este proceso a la comunidad, motivaron e implementan acciones con las recomendaciones surgidas de la situación encontrada inicialmente, con el propósito de lograr estados superiores de desarrollo. Así, se logra el empoderamiento de la comunidad, participando activamente en la toma de decisiones sobre ¿qué camino seguir? y ¿qué hacer en cada momento? Esto implica el desarrollo de un modelo de organización social sustentado en la participación, con un trabajo conjunto entre facilitadores y población intervenida para lograr desarrollos coherentes con sus necesidades, la autonomía, el liderazgo, responsabilidad y gestión de las 
emociones que se deben abordar entre otros temas en la capacitación, donde la familia es parte activa para lograr interiorizar a nivel comunitario la importancia de la subsistencia a través del trabajo y las relaciones de convivencia pacífica.

En cuanto al conocimiento de organizaciones existentes en el barrio, en la tabla No. 7 se presentan los resultados. El 90 \% manifiesta conocerlas, como son la asociación de personas de la tercera edad, organismo integrador, Fundación Revivir SigloXXI, en las que se desarrollan programas de integración de la población, capacitación, prácticas agrícolas y esparcimiento para el mejoramiento de la salud y utilización del tiempo libre, entre otros.

\begin{tabular}{|l|l|}
\hline Respuesta & $\%$ \\
\hline Si & 90 \\
\hline No & 10 \\
\hline TOTAL & 100 \\
\hline
\end{tabular}

Tabla 7. Conocimiento de Organizaciones Comunitarias existentes en el Barrio Loma Linda.

Fuente. Elaboración propia. Investigación 2016. Encuesta aplicada a la población.
Igualmente, conocen organizaciones de carácter religioso en donde los Dominicos a través de Universidad Santo Tomás, con el Centro de Proyección Social y con la Capilla de Santa María de los Ángeles, ubicada en cercanías de la Fundación mencionada, reciben orientaciones para la formación de vida y del trabajo. La comunidad religiosa de los padres Eudistas, de la Universidad Minuto de Dios, quienes adelantan programas de recreación y actividades lúdicas para grupos de población de diferentes edades. El 10\% restante manifiestan no tener conocimiento de las organizaciones sociales existentes en la localidad, respuesta no congruente por cuanto todos los participantes se enriquecen de los programas de la Fundación Revivir Siglo XXI, como organismo integrador de las entidades que la benefician.

En la fotografía No.2 se puede observar los talleres de participación e integración de la comunidad, en los cuales suministraron suficiente información acerca de las intenciones de organizarse para el desarrollo de las actividades productivas de la agricultura urbana.

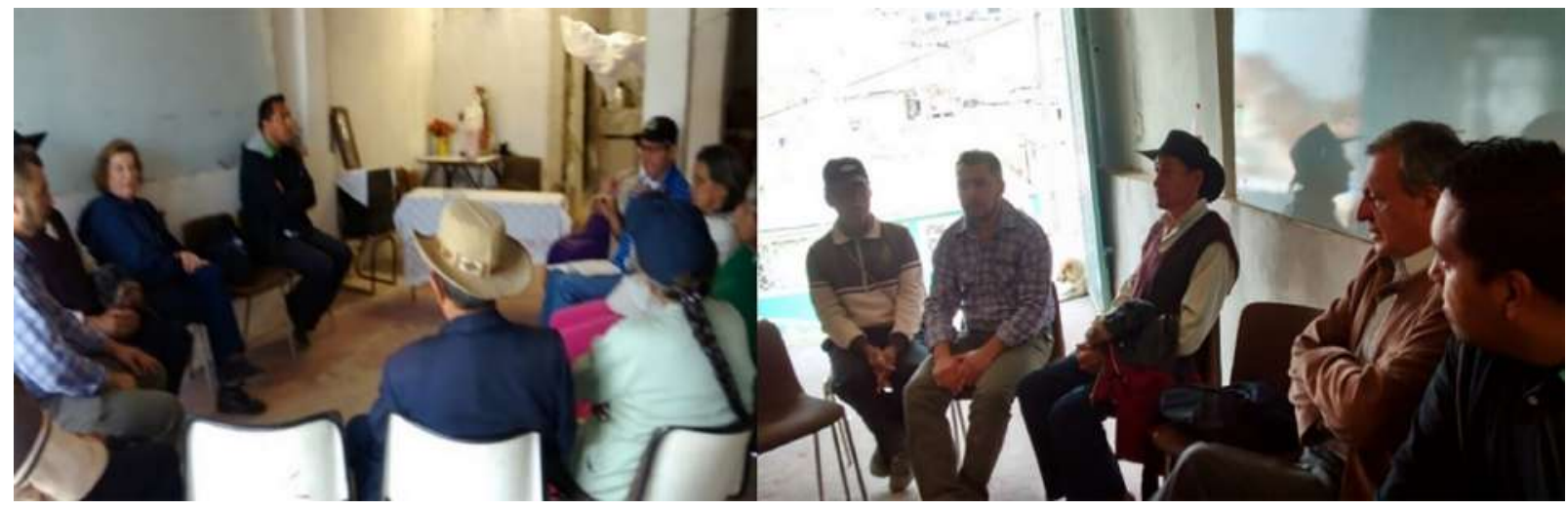

Fotografía 2. Reuniones con la comunidad en la Asociación de la tercera edad en Altos de Cazucá.

Fuente. Elaboración propia. 
Como resultado de estos talleres se obtuvo la información que se registran en la tabla No.8. Como se observa, el $40 \%$ de esta población manifestó la importancia de asociarse para constituir una organización de carácter empresarial, mientras que el 30\% demostró dificultades y apatía para participar en ella.

\begin{tabular}{|l|l|}
\hline Respuesta & $\%$ \\
\hline SI & 40 \\
\hline NO & 30 \\
\hline NO SABE & 30 \\
\hline TOTAL & 100 \\
\hline
\end{tabular}

Tabla 8. Constituir organización comunitaria.

Fuente. Elaboración propia. Investigación 2016. Encuesta aplicada a la población.

El 30\% responden que no saben o no son conscientes de la importancia de esta iniciativa, bien por la limitación de recursos o apatía social a los procesos de integración en actividades productivas.

La formación de las personas que integran la comunidad no se limitó a la adquisición de algunos conocimientos y técnicas para la realización del trabajo, sino que se desarrolló el análisis y reflexión sobre su propia realidad y sobre las actividades que van a realizar.
Se adelantó un proceso educativo con la comunidad, en el que se propuso la generación de nuevos valores, nuevas formas de integración de la comunidad e interacción con la sociedad, mediante el diálogo, formas de comunicación que sustituyan actitudes como el individualismo, la falta de solidaridad, la indiferencia, la intolerancia y el egoísmo que obstaculiza la integración y la organización comunitaria. Así, la capacitación fue importante, para desarrollar actitudes, capacidades y habilidades en la dirección y organización de un trabajo en equipo cooperado, con esfuerzo mancomunado y que no todos estaban en condiciones de emprender.

En el componente de organización comunitaria se adelantaron seis (6) talleres en los que participaron en promedio 40 familias, se enfatizó en valores de comportamiento para la convivencia familiar, ciudadana y organizacional, y enfocados hacia valores de autoayuda (actividad, creatividad, responsabilidad de independencia), valores de ayuda mutua (cooperación, unidad de acción colectiva, solidaridad y paz), valores sin ánimo de lucro (conservación de recursos, eliminación de las ganancias como móviles dominantes, responsabilidad social y metas útiles), en concordancia con los temas tratados por Ramirez (1989, p.136) abordados por Marcus Lars y descritos en el marco teórico.

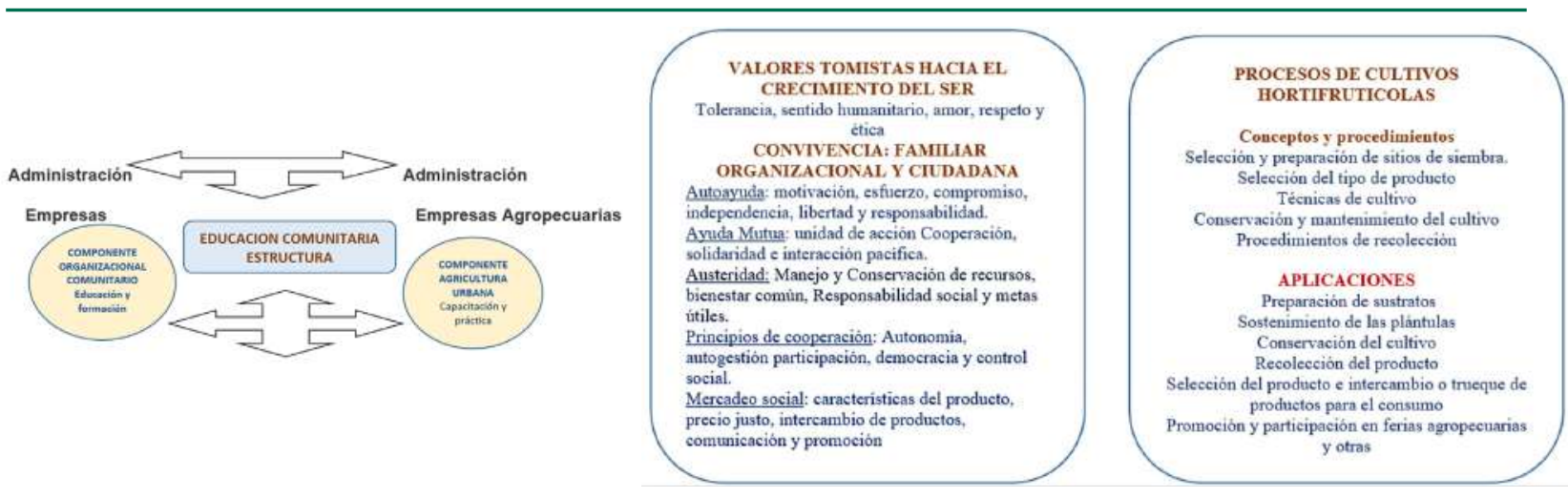

Figura 1. Enfoque de la capacitación comunitaria en poblaciones vulnerables en Loma Linda, Altos de Cazucá, de acuerdo a programa académico participante en el proyecto de investigación y semillero de investigación.

Fuente. Elaboración propia. 
Con el pensamiento Tomista se trataron los valores y virtudes del ser humano, como tolerancia, sentido humanitario, amor, respeto y ética, entre otros. En cuanto a principios se cubrieron temas relacionados con la autogestión, participación y democracia que dan las bases para la asociatividad de interés y recurso comunes.

En cuanto al componente de agricultura urbana, en los talleres interactivos, el 65\% de las personas manifestaron la necesidad de recibir capacitación en las actividades de producción agrícola urbana, desde el proceso de selección de material de siembra, preparación de los sustratos y sostenimiento de la plántulas o cultivos en su fase de desarrollo, hasta los sistemas de recolección, clasificación, empaque y venta de los productos y subproductos a obtener.

\begin{tabular}{|l|l|}
\hline Respuesta & $\%$ \\
\hline SI & 65 \\
\hline NO & 25 \\
\hline TOTAL & 90 \\
\hline
\end{tabular}

Tabla 9. Capacitación en Agricultura Urbana.

Fuente. Elaboración propia. Investigación 2016. Encuesta aplicada a la población.

El 25\% restante manifestaron no estar interesados en estos procesos de capacitación (Tabla No. 9), aunque en el desarrollo de los talleres respectivos participó la mayor parte de ellos, ver fotografía No.3.

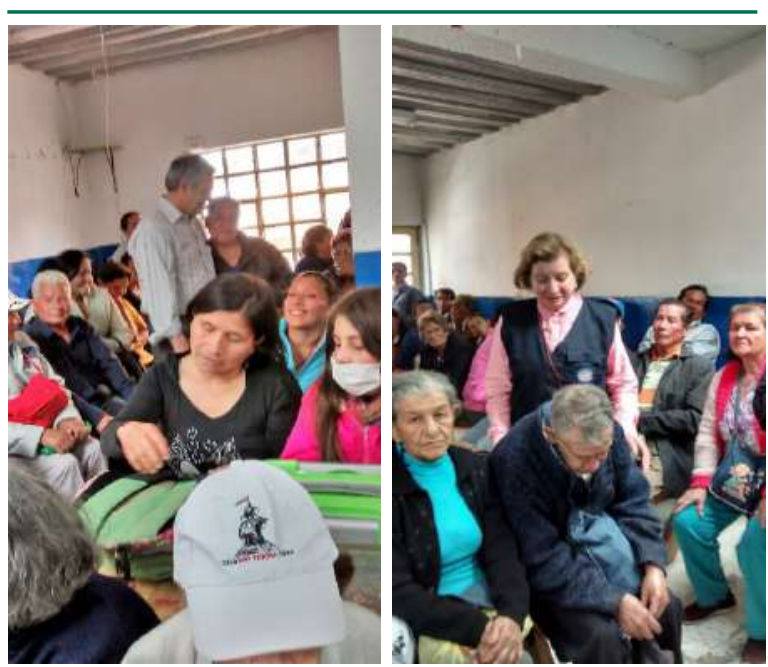

Fotografía 3. Taller interactivo con comunidad.

Fuente. Elaboración propia.

El componente de agricultura urbana, fue adelantado por el Semillero de Investigación denominado Transformadores de la realidad, del programa de Administración de Empresas Agropecuarias, a través de talleres prácticos desarrollados los días sábados con el acompañamiento de los estudiantes y del coordinador del Semillero.

En tales talleres, se abordaron los temas de sistemas de producción, preparación del material de siembra (selección y desinfección de semilla, pre-germinado, germinado), sustratos de siembra, trasplante o siembra, fertilización, controlde plagas y enfermedades y cosecha de productos, trabajo desarrollado por los miembros de la comunidad en los espacios físicos dispuestos por ellos mismos y utilizando sus propios recursos.

Así, se llevó a cabo la I Feria Agrícola Urbana, en las instalaciones de la Fundación Revivir Siglo XXI, en la que participaron familias con muestras y productos logrados por ellos mismos. También se demostró el sentido de pertenencia y solidaridad del grupo al trabajar de manera cooperada. 
Una desventaja observada en estos programas de capacitación, fue la participación de un grupo mayoritario de la tercera edad que no garantiza la sostenibilidad o continuidad de un proceso productivo de agricultura urbana en la que se pueda conseguir la especialización productiva por especie y manejo escalonado de sistemas de siembra, para regular los picos de cosecha y obtener una producción en todas las épocas del año, que facilite el abastecimiento continuo y una estabilidad de precios.

\section{Impactos de la investigación}

Se logró la motivación de la comunidad para el desarrollo de actividades productivas del componente hortofrutícola, de manera cooperada y solidaria, con lo que se fortaleció la experiencia individual, especialmente de la población desplazada de zonas rurales, dando un impulso al inicio de cultivos de verduras como la espinaca, lechuga, acelgas, zanahorias tomate y frutas como la curuba y la mora.

Desarrollaron la innovación y creatividad para involucrar los espacios de la vivienda como medio para generar cultivos en diferentes formas y recipientes que facilitó obtener productos para el consumo familiar. De igual forma, les permitió realizar intercambio de productos y la venta de algunos excedentes entre ellos mismo, con lo que lograron un ingreso para cubrir otras necesidades.

La experiencia sirvió para crear la feria agropecuaria en Altos de Cazucá, como instrumento para el mercadeo y promoción de productos en la localidad, lo cual generó satisfacción y bienestar al reconocer la capacidad de trabajo de la población adulta mayor (tercera edad), y el uso del tiempo libre en actividades benéficas para la salud del cuerpo y mente.
Para la Universidad se recogen elementos importantes para el desarrollo de programas de agricultura urbana, en los que no sólo cuenta la técnica, sino el trabajo de motivación de las personas que por diversas circunstancias se encuentran en pobreza extrema.

Los principios y valores humanistas de la filosofía Tomista, permitieron encauzar los procesos de formación del comportamiento y desarrollo de actitudes hacia la asociatividad para el logro de intereses comunes.

\section{Conclusiones}

La condición de desplazamiento de zonas rurales, es una oportunidad para aprovechar las experiencias de la población en actividades agrícolas y pecuarias, en procesos productivos, bajo condiciones de agricultura urbana.

Se evidencia la prevalencia del adulto mayor con más de 60 años, que, por su capacidad de trabajo y condiciones de aprendizaje, puede limitar las actividades productivas de la agricultura urbana con tecnologías más avanzadas y desarrollar proyectos sostenibles de mediano largo plazo. Se establece la importancia de vincular población joven en los procesos de agricultura urbana

Se evidenció la importancia de los productos hortofrutícolas en la base alimentaria de la población vulnerable en altos de Cazucá, y en consecuencia el interés de la comunidad en desarrollar proyectos productos en la modalidad de agricultura urbana, para suplir la necesidad y generar excedentes comercializables en intercambio con los mismos miembros de la comunidad o venta directa en la localidad.

El costo promedio de los productos hortofrutícolas seleccionados para el estudio 
asciende a un valor total por mes de $\$ 154.100$ pesos, lo cual equivale a $\$ 25.683$ per cápita. Esto indica que cada familia dedica el $61.2 \%$ de sus ingresos para adquirir tales productos, cuando el promedio de la población pobre de Bogotá dedica cuanto menos el $35 \%$ de sus ingresos.

Se observa un alto porcentaje de la población adulta (70\%) sin vínculo laboral, que desempeña alguna actividad independiente en su entorno o en su propia vivienda, generadora de ingresos poco remunerados. Entre las actividades que más se desarrollan estáel cuidado de niños, que generalmente son de su mismo núcleo familiar (nietos, sobrinos) o hijos de vecinos.

El $72 \%$ de las familias tienen ingresos que se ubica en el rango entre $\$ 101.000$ y $\$ 400.000$ pesos, para un promedio ponderado de $\$ 252.000$ por mes. El 64\% de la población recibe ingresos inferiores a $\$ 300.000$ mensuales y el $15 \%$ obtienen menos de $\$ 100.000$, con lo que se demuestra que esta zona de Cazucá se encuentra en condiciones de pobreza extrema, por cuanto este valor se encuentra por debajo de lo establecido por el gobierno nacional que es del orden de los $\$ 211.807$ pesos mensuales.

La comunidad consideró importante la capacitación práctica recibida del Semillero de investigación en los procesos de la agricultura urbana y la participación en las ferias de la fundación y del emprendimiento en la Universidad San Tomás, con muestras representativas de sus productos obtenidos en sus proyectos.

Resalta la importancia que tiene el Centro de Proyección Social de la Universidad Santo Tomás en Altos de Cazucá, por el conocimiento de las diversas poblaciones que conforman los barrios de esta Comuna, y la influencia que ejerce en el desarrollo de los proyectos de proyección social, que como este coadyuvan a lograr un mejor bienestar y desarrollo comunitario. Así mismo, se manifiesta el nivel de integración de este centro con la Fundación Revivir Siglo XXI y la Universidad del Minuto de Dios para el desarrollo de actividades conjuntas con el propósito de mitigar en alguna medida las condiciones críticas en las que vive la comunidad.

\section{REFERENCIAS}

Bernal,A. (2006). Metodología de la investigación para administración, economía, humanidades y ciencias sociales. México: Pearson..

Brito, A., Betancourt, M., \& Hernández, J. (2012). Seguridad y Soberanía Alimentaria. Recuperado de: http://www. monografias.com/trabajos93/seguridad-y-soberania-alimentaria/seguridad-y-soberania-alimentaria2.shtml\#ixzz41ELV9MFe

Burgers, A. \& Glasauer, P. (2006). Guía de nutrición de la familia. Organización de las Naciones Unidas para la Agricultura y la Alimentación. Recuperado de:

ftp://ftp.fao.org/docrep/fao/oo9/y5740s/ y5740s.pdf

Burgos, F. (2007). Estudio de factibilidad para creación de microempresa de cultivo de plantas alimenticias en espacios urbanos en la localidad Antonio Nariño en Bogotá a partir de proyecto de Agricultura Urbana del Plan de Desarrollo "Bogotá sin Indiferencia". (Trabajo monográfico de especialización, Universidad de la Salle). Recuperado de: http://repository.lasalle. edu.co/handle/10185/2214 .

Concejo Municipal de Soacha. (27 de diciembre del 200o). Plan de Ordenamiento Territorial. [Acuerdo No. 46 del 
2000]. Recuperado de: http://www. observatorioambientalcar.co/archivos/1393367993pot.pdf

Fundación Eroski. (2016). Eroski Consumer. Hortaliza y verduras. Guía práctica de verduras. Recuperado de: http://verduras. consumer.es/

Gómez, J. (2014). Agricultura urbana en América Latina y Colombia. (Trabajo monográfico, Universidad Nacional Abierta y a Distancia- UNAD). Recuperado de: http://repository.unad.edu.co/bitstream/10596/2749/1/15385851.pdf

Hermi-Zaar, M. (2011). Agricultura urbana. Reflexiones sobre su origen e importancia actual. Revista Bibliográfica de Geografía y Ciencias Sociales, XVI(944). Recuperado de: http://www.ub.edu/geocrit/b3w-944. htm

Hernández S., Fernández, C. \& Baptista, L. (2006), Metodología de la investigación. México: McGraw-Hill Interamericana.

Jardín Botánico "José Celestino Mutis". (2007). Cartillas Técnicas. Agricultura Urbana. Disponible en: https://es.scribd. com/doc/31695652/CARTILLA-TECNICA-AGRICULTURA-URBANA- JARDIN-BOTANICO

Mosquera, J. (2009). Efectos socioeconómicos y ambientales de la agricultura urbana caso: unidades de planeamiento zonal (upzs) de Rincón y Tibabuyes integradas, localidad de suba, Bogotá, D.C. (Tesis de maestría, Pontificia Universidad Javeriana). Recuperado de: https://repository. javeriana.edu.co/handle/10554/744

Organización de las Naciones Unidas para la Agricultura y la Alimentación, FAO. (1999) Cuestiones de la agricultura urbana. Enfo- ques, (21). Recuperado de: http://www.fao. org/Ag/esp/revista/9901sp2.htm

Organización de las Naciones Unidas para la Agricultura y la Alimentación, FAO. (2010). División de Comercio y Mercados. Programa de la FAO para la horticultura urbana y periurbana (HUP), División de Producción y Protección Vegetal. Organización de las Naciones Unidas para la Agricultura y la Alimentación. Viale delle Terme di Caracalla o0153 Roma, Italia.

Prakash, A. (2010) Horticultura urbana y periurbana: Impacto en la seguridad alimentaria, la economía y el empleo. Recuperado de: http://www.fao.org/ag/agp/greenercities/pdf/HD/HUP-HD-1.pdf

Ramírez, B. (1989). Teoría y doctrina de la cooperación. Bogotá: Arfo Editores Ltda.

Restrepo, B., Sierra,J. \& Bayona.M. (2014). La empresa de economía solidaria, una alternativa de solución para comunidades vulnerables en el municipio de Soacha, comuna IV, Altos de Cazucá. Revista Arbitrada del Centro de Investigación y Estudios Gerenciales (4), 144 -159.

Robles, M. Comisión Económica para el desarrollo de América Latina y del Caribe, CEPAL. Determinación del ingreso familiar, encuesta integrada de hogares. Secretaría Técnica de Planificación del Desarrollo Económico y Social, Dirección General de Estadística, Encuestas y Censos 19971998, Paraguay.iábato, M. (2009). Lineamientos metodológicos para la educación solidaria. Bogotá: Universidad Cooperativa de Colombia.

Sierra, B. \& Peña, M. (2014). Trabajo Comunitario. Bogotá: Universidad Santo Tomás-VUAD. 